\title{
Evaluación del grado de acidez, flujo salival y placa dental en gestantes y no gestantes, Hospital Regional Virgen de Fátima, Chachapoyas - 2018
}

\section{Evaluation of the degree of acidity, salivary flow and dental plaque in pregnant and non- pregnant women at the Virgen de Fatima Regional Hospital, Chachapoyas - 2018}

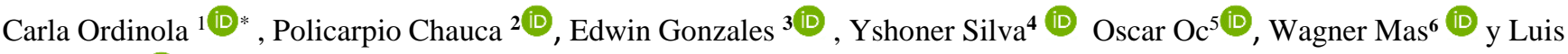 \\ Gutierrez $^{7}$ (i)
}

\section{RESUMEN}

La presente investigación de enfoque cuantitativo; nivel relacional comparativo; tipo observacional, prospectivo, transversal y analítico; evaluó el grado de acidez, flujo salival y placa dental de pacientes gestantes y no gestantes, Hospital Regional Virgen de Fátima, Chachapoyas - 2018. Así, 118 mujeres (60 gestantes y 58 no gestantes) fueron atendidos en el HRVF, Chachapoyas - Amazonas. Se midió el pH salival con un pH-metro digital Oakton pH 450, se recolectó saliva segregada no estimulada, medido en jeringas de tuberculina, la placa dental se evidenció al administrar una pastilla reveladora, anotando los valores observados en la ficha para su posterior cálculo del Índice de Higiene Oral Simplificado (IHOS). Obteniendo el $5.1 \%$ presentó pH ácido y el $44.1 \%$ presentó pH neutro (no gestantes); $20.4 \%$ presentó pH ácido y el $30.5 \%$ presentó pH neutro (gestantes). Flujo salival, ambos grupos presentan un nivel muy alto, solo el $2.5 \%$ se encuentra en un rango normal en no gestantes y $0.8 \%$ se encuentra en un nivel bajo en gestantes. El (IHOS), el $11.9 \%$ y el $29.7 \%$ se encuentran en parámetros de óptimo y regular respectivamente, el 7.6\% se encuentra en un parámetro malo (no gestantes). El $0.8 \%$ optimo, el $25.4 \%$ regular, el $23.7 \%$ en malo y el $0.8 \%$ muy malo (gestantes).

Palabras clave: $\mathrm{pH}$, flujo salival, saliva, placa dental, gestación, hospital.

\begin{abstract}
The present research of quantitative approach; comparative relational level; Observational, prospective, transversal and analytical type; assesses the degree of acidity, salivary flow and dental plaque of pregnant and non-pregnant patients, HRVF, Chachapoyas - 2018. Thus, 118 women (60 pregnant women and 58 non-pregnant women) attended at the HRVF, Chachapoyas - Amazonas. Salivary pH was measured with an Oakton $\mathrm{pH} 450$ digital $\mathrm{pH}$ meter, segregated unstimulated saliva was collected for 5 minutes, measured in tuberculin syringes, dental plaque was evident when administering a revealing tablet, writing down the values observed in the card for its subsequent calculation of (IHOS). Obtaining $5.1 \%$ had an acid $\mathrm{pH}$ and $44.1 \%$ had a neutral $\mathrm{pH}$ (not pregnant); $20.4 \%$ had an acid $\mathrm{pH}$ and $30.5 \%$ had a neutral $\mathrm{pH}$ (pregnant). Salivary flow, both groups present a very high level, only $2.5 \%$ are in a normal range in nonpregnant women and $0.8 \%$ are in a low level in pregnant women. In (IHOS), $11.9 \%$ and $29.7 \%$ are in optimal and regular parameters respectively, $7.6 \%$ are in a bad parameter (non-pregnant). The optimal $0.8 \%$, the regular $25.4 \%$, the $23.7 \%$ in bad and the $0.8 \%$ very bad (pregnant).
\end{abstract}

Keywords: pH, salivary flow, saliva, dental plaque, pregnancy, hospital.

DOI: https://doi.org/10.37787/pakamuros-unj.v8i3.134

Recibido: 15/07/2020. Aceptado: 09/10/2020

* Autor para correspondencia

1. Universidad Nacional Toribio Rodríguez. de Mendoza de Amazonas, Perú. Email: carla.ordinola@ untrm.edu.pe 2. Email: policarpio.chauca@untrm.edu.pe 3. Email: edwin.gonzales@untrm.edu.pe 4. Email: yshoner.silva@untrm.edu.pe 5. Email: oscar.oc@untrm.edu.pe 6. Email: wagner.mas@untrm.edu.pe 7. Email: lgutierrezberru@gmail.com 


\section{INTRODUCCIÓN}

La gestación es un periodo en la vida de la mujer sexualmente activa, más importante, donde el óvulo fecundado en su cuerpo se desarrolla hasta formar el feto, que nace después de completar su crecimiento y maduración siendo aproximadamente a los 280 días (9 meses o 40 semanas). Durante el proceso de gestación se da una serie de cambios en el organismo de la madre para poder prepararla tanto a ella como para el desarrollo de su futuro bebé. Aunado a esos cambios fisiológicos se originan también alteraciones en la boca de la embarazada. Si bien el embarazo constituye una condición sistémica particular que modifica las condiciones bucodentales, el diente además de la mucosa bucal, los tejidos de soporte y sostén, entre otros, constituyen blancos directos que pueden afectarse por este motivo debido a que la gestante está sometida a una serie de cambios extrínsecos e intrínsecos relacionados entre sí haciéndola vulnerable a padecer alguna patología (Cerna, 2010. p. 40 - 41).

En diversas investigaciones realizadas a nivel nacional concerniente a las enfermedades que aquejan a las gestantes o no gestantes; se pueden rescatar que en gran mayoría son problemas bucales por lo que la saliva es un líquido de la cavidad bucal, transparente, de viscosidad variable, compuesto principalmente por agua, sales minerales y algunas proteínas, al disminuir los niveles de flujo disminuye su capacidad protectora del medio bucal, la neutralización de los ácidos y la limpieza de los restos alimenticios, aumentando el riesgo de presentar gingivitis, enfermedades periodontales y xerostomía. El Potencial Hidrógeno $(\mathrm{pH})$, es un parámetro muy usado en química para medir el grado de acidez o alcalinidad de las sustancias. Es un factor clave para que muchas reacciones. El pH está regulado por la saliva, sus valores normales oscilan entre los 6.5 y 7 (Delgado, 2017. p. 25). Por lo tanto, es neutro y debido a su contenido de bicarbonato tiene propiedades neutralizantes de los ácidos, jugando un importante papel en la higiene de la boca. Durante el embarazo las mujeres experimentan varios cambios hormonales que podrían afectar el flujo salival y el $\mathrm{pH}$ siendo de este modo factores causales del aumento de enfermedades de la cavidad oral (Chaupis, 2016. p. 34). La saliva es una secreción exocrina compleja, importante en el mantenimiento de la homeostasis de la cavidad bucal.

El gobierno peruano por medio del Ministerio de Salud toma en cuenta el proceso gestacional, el postparto y el crecimiento y desarrollo del nuevo ser, sin embargo en la región Amazonas es común ver mujeres gestantes y/o lactantes con múltiples problemas de salud oral lo que nos deja imaginar que probablemente durante el proceso gestacional existen cambios en el medio bucal (variaciones del $\mathrm{pH}$, flujo salival, etc.) repercutiendo en la flora bacteriana y estas en múltiples problemas de salud oral, razón por la cual se realizó la presente investigación. 


\section{MATERIALES Y MÉTODOS}

\section{Diseño de investigación}

La presente investigación tuvo enfoque cuantitativo; de nivel relacional comparativo; de tipo: observacional; según la planificación de la toma de datos fue prospectivo; según el número de ocasiones en que se midió la variable de estudio fue transversal y según el número de variables de interés fue analítico (Supo, 2015, pp. 2 - 19).

De enfoque cuantitativo porque permitió cuantificar los datos mediante el uso de la estadística. De nivel descriptivo porque describió los hechos tan igual como sucede en la naturaleza. Tipo de investigación fue observacional porque no se manipularon las variables ya que los datos reflejaron la evolución natural de los eventos. Prospectivo porque los datos se recolectaron de fuentes primarias o sea directamente de la muestra objeto de estudio. Transversal porque las variables se midieron en una sola ocasión. Analítico porque el análisis estadístico fue trivariado y permitió contrastar las hipótesis de ambas muestras de estudio. Además, el diseño de investigación fue relacional comparativo.

\section{Población, muestra y muestreo}

a) Universo

Fue conformado por 118 mujeres atendidas en el tercer trimestre 2018 en el Hospital Regional Virgen de Fátima, Chachapoyas - Amazonas.

b) Población.

De acuerdo a los criterios de inclusión y exclusión la población estaba constituida por 118 mujeres atendidas en el Hospital Regional Virgen de Fátima, Chachapoyas - 2018.

c) Muestra.

Estuvo constituida por el $100 \%$ de la población (60 gestantes y 58 no gestantes que se atienden en el Hospital regional Virgen de Fátima, Chachapoyas - Amazonas.

d) Muestreo

Se utilizó el muestreo probabilístico aleatorio simple, que consistió en colocar en una bolsa el total de balotas como casos de la población de estudio enumerados desde el 1 al 24, de los cuales la primera balota se consideró para el grupo experimental, la segunda para el grupo control, la tercera para el grupo experimental y así sucesivamente hasta que se completó el total de las unidades muestras de estudio en ambos grupos tanto experimental como el grupo control.

\section{Métodos de Investigación}

Durante el proceso de investigación, se empleó el método científico Inductivo - deductivo e hipotético - deductivo. 


\section{Técnicas e instrumentos de recolección de datos}

Se realizó mediante la técnica de la documentación, y para recabar la información se utilizó:

$\mathrm{V} 1=\mathrm{pH}-$ metro Oakton $\mathrm{pH} 450$, el cual nos contrastó los resultados:

\begin{tabular}{|l|c|c|c|}
\hline $\mathrm{pH}-$ Salival & Ácido $\leq 6$ & Neutro 7 & Alcalino $\geq 8$ \\
\hline
\end{tabular}

V2 = Jeringa de Tuberculina, el cual brindó los siguientes resultados:

\begin{tabular}{|l|c|c|c|}
\hline Flujo Salival & $\begin{array}{c}\text { Bajo } \\
\text { Menor a } 0.3 \mathrm{ml}\end{array}$ & $\begin{array}{c}\text { Neutro } \\
0.3-0.5 \mathrm{ml}\end{array}$ & $\begin{array}{c}\text { Alcalino } \\
\text { Mayor a } 0.5 \mathrm{ml}\end{array}$ \\
\hline
\end{tabular}

V3 = IHOS, el cual brindó los siguientes resultados:

\begin{tabular}{|l|c|c|c|c|}
\hline Placa dental & Óptimo & Regular & Malo & Muy malo \\
& De 0.0 a 1.0 & De 1.1 a 2.0 & De 2.1 a 3.1 & Mayor a \\
& puntos & Puntos & puntos & 3.1 puntos \\
\hline
\end{tabular}

\section{Materiales e Instrumentos.}

- Uniforme y/o mandil.

- Mascarilla.

- Guantes.

- Vasos descartables.

- Jeringas de tuberculina (1ml).

- $\mathrm{pH}$ - metro Oakton $\mathrm{pH} 450$

- Pastillas reveladoras de placa.

- Cepillos y pasta dental.

- Papel toalla.

- Espejos Intraorales.

- Ficha de registro de pH salival, Flujo Salival e IHOS.

- Lapiceros azul o negro.

- Líquidos para calibrar $\mathrm{pH}$ - metro.

- Agua destilada.

\section{Procedimiento.}

Se hizo a través de los pasos:

- Se solicitó autorización al Director del Hospital Regional Virgen de Fátima Chachapoyas.

- Obtenido el permiso, se procedió a fijar fecha, día y hora en la que se realizó la recolección de los datos.

- Se solicitó el permiso a las mujeres gestantes y no gestantes que acuden al Hospital. 
- Se revisaron las cavidades orales de las mujeres gestantes y no gestantes que acuden al Hospital Regional Virgen de Fátima y se registraron los datos de acuerdo a los criterios establecidos, en función de las variables en estudio.

- En caso de las gestantes se anotó el periodo de gestación en el que se encontraban, para identificar el trimestre con mayor diferencia en las variables.

- Los datos obtenidos en las fichas se revisaron y sin datos faltantes se pasaron al software Excel de manera ordenada e independiente de ambos grupos de estudio.

- Los datos obtenidos se tabularon con el apoyo de paquetes estadísticos (SPSS.25) para su análisis estadístico.

- Finalmente se hizo un análisis de los resultados encontrados

\section{RESULTADOS}

Tabla 1. pH salival, flujo salival y placa dental en gestantes y no gestantes del hospital regional Virgen de Fátima, Chachapoyas - 2018 .

\section{Edad gestacional}

\begin{tabular}{|c|c|c|c|c|c|c|c|c|c|c|c|}
\hline \multirow[t]{2}{*}{ Variable } & \multirow[t]{2}{*}{ Parámetro } & \multicolumn{2}{|c|}{ No gestante } & \multicolumn{2}{|c|}{$\begin{array}{l}\text { De } 1 \text { a } 14 \\
\text { semanas }\end{array}$} & \multicolumn{2}{|c|}{$\begin{array}{r}\text { De } 15 \text { a } 28 \\
\text { Semanas }\end{array}$} & \multicolumn{2}{|c|}{$\begin{array}{r}\text { De } 29 \text { a } 42 \\
\text { Semanas }\end{array}$} & \multicolumn{2}{|c|}{ Total } \\
\hline & & fi & $\%$ & $\mathrm{Fi}$ & $\%$ & $\mathrm{Fi}$ & $\%$ & fi & $\%$ & $\mathrm{Fi}$ & $\%$ \\
\hline \multirow{3}{*}{$\mathrm{pH}$} & Ácido & 6 & $5,1 \%$ & 0 & $0,0 \%$ & 6 & $5,1 \%$ & 18 & $15,3 \%$ & 30 & $25,4 \%$ \\
\hline & Neutro & 52 & $44,1 \%$ & 4 & $3,4 \%$ & 19 & $16,1 \%$ & 13 & $11,0 \%$ & 88 & $74,6 \%$ \\
\hline & Total & 58 & $49,2 \%$ & 4 & $3,4 \%$ & 25 & $21,2 \%$ & 31 & $26,3 \%$ & 118 & $100,0 \%$ \\
\hline \multirow{5}{*}{$\begin{array}{c}\text { Flujo } \\
\text { Salival }\end{array}$} & Bajo & 0 & $0,0 \%$ & 0 & $0,0 \%$ & 0 & $0,0 \%$ & 1 & $0,8 \%$ & 1 & $0,8 \%$ \\
\hline & Normal & 3 & $2,5 \%$ & 0 & $0,0 \%$ & 0 & $0,0 \%$ & 0 & $0,0 \%$ & 3 & $2,5 \%$ \\
\hline & Alto & 55 & $46,6 \%$ & 4 & $3,4 \%$ & 25 & $21,2 \%$ & 30 & $25,4 \%$ & 114 & $96,6 \%$ \\
\hline & Total & 58 & $49,2 \%$ & 4 & $3,4 \%$ & 25 & $21,2 \%$ & 31 & $26,3 \%$ & 118 & $100,0 \%$ \\
\hline & optimo & 14 & $11,9 \%$ & 0 & $0,0 \%$ & 1 & $0,8 \%$ & 0 & $0,0 \%$ & 15 & $12,7 \%$ \\
\hline \multirow{4}{*}{ IHOS } & regular & 35 & $29,7 \%$ & 4 & $3,4 \%$ & $\begin{array}{l}1 \\
5\end{array}$ & $12,7 \%$ & 11 & $9,3 \%$ & 65 & $55,1 \%$ \\
\hline & malo & 9 & $7,6 \%$ & 0 & $0,0 \%$ & 8 & $6,8 \%$ & $\begin{array}{l}2 \\
0\end{array}$ & $16,9 \%$ & 37 & $31,4 \%$ \\
\hline & muy malo & 0 & $0,0 \%$ & 0 & $0,0 \%$ & 1 & $0,8 \%$ & 0 & $0,0 \%$ & 1 & $0,8 \%$ \\
\hline & Total & 58 & $49,2 \%$ & 4 & $3,4 \%$ & 25 & $21,2 \%$ & 31 & $26,3 \%$ & 118 & $100,0 \%$ \\
\hline
\end{tabular}

En la Tabla 1, se observa que, 118 pacientes mujeres accedieron a participar de la investigación, de las cuales, en relación al pH salival, el $44.1 \%$ que presento $\mathrm{pH}$ neutro fueron no gestantes y solo el $5.1 \%$ presentaron pH ácido. El $20.4 \%$ que presentó pH ácido y el $30.5 \%$ pH neutro, eran gestantes. Respecto 
al flujo salival, el $46.6 \%$ que presentó un flujo salival alto eran no gestantes, solo $2.5 \%$ se presentó en normal; el $50 \%$ que presentó flujo salival alto eran gestantes y solo $0.8 \%$ se encontró en un rango bajo. En caso de la presencia de placa dental de las no gestantes, el $11.9 \%$ se encuentra en un índice óptimo, el $29.7 \%$ en regular, el $7.6 \%$ en malo; de las gestantes el $0.8 \%$ se encuentra en índice óptimo, el 25.1 $\%$ en regular, el $23.7 \%$ en malo y solo el $0.8 \%$ es muy malo.

Tabla 2. pH salival y placa dental en gestantes y no gestantes del hospital regional Virgen de Fátima, Chachapoyas - 2018 pH salival

IHOS

Total

\begin{tabular}{ccccccccccc} 
& \multicolumn{2}{c}{ Optimo } & \multicolumn{2}{c}{ Regular } & \multicolumn{2}{c}{ Malo } & \multicolumn{2}{c}{ Muy malo } & \\
\cline { 2 - 9 } & fi & $\%$ & fi & $\%$ & fi & $\%$ & fi & $\%$ & fi & $\%$ \\
\hline Acido & 0 & $0.0 \%$ & 4 & $3.4 \%$ & 26 & $22.0 \%$ & 0 & $0.0 \%$ & 30 & $25.4 \%$ \\
Neutro & 15 & $12.7 \%$ & 61 & $51.7 \%$ & 11 & $9.3 \%$ & 1 & $0.8 \%$ & 88 & $74.6 \%$ \\
\hline Total & 15 & $12.7 \%$ & 65 & $55.1 \%$ & 37 & $31.4 \%$ & 1 & $0.8 \%$ & 118 & $100.0 \%$ \\
\hline
\end{tabular}

Fuente: Ficha de recolección de datos

En la Tabla 2, podemos observar que del $73.5 \%$ de pacientes que presentaron $\mathrm{pH}$ neutro, el $12.7 \%$ se presentó en un índice óptimo, el $51.7 \%$ en índice regular, el $9.3 \%$ en malo y el $0.8 \%$ en muy malo; del $25.4 \%$ que presentaron pH ácido, el $3.4 \%$ presentaron un índice regular y el $22 \%$ índice malo.

La ji cuadrada $=57,433 ; \mathrm{gl}=3 ; \mathrm{p}=0.000<\alpha=0.05$. Nos indica que existe una relación estadísticamente significante entre el $\mathrm{pH}$ salival y la placa dental en gestantes y no gestantes.

Tabla 3. pH entre gestantes y no gestantes del hospital regional Virgen de Fátima, Chachapoyas - 2018

\section{Edad Gestacional}

\begin{tabular}{|c|c|c|c|c|c|c|c|c|c|c|}
\hline $\begin{array}{c}\text { pH } \\
\text { salival }\end{array}$ & & $\begin{array}{c}\text { No } \\
\text { gestante }\end{array}$ & & $\begin{array}{c}\text { De } 1 \text { a } 14 \\
\text { semanas }\end{array}$ & & $\begin{array}{c}\text { De } 15 \text { a } 28 \\
\text { semanas }\end{array}$ & & $\begin{array}{l}9 \text { a } 42 \\
\text { manas }\end{array}$ & & Total \\
\hline & fi & $\%$ & fi & $\%$ & fi & $\%$ & fi & $\%$ & fi & $\%$ \\
\hline Acido & 6 & $5.1 \%$ & 0 & $0.0 \%$ & 6 & $5.1 \%$ & 18 & $15.3 \%$ & 30 & $25.4 \%$ \\
\hline Neutro & 52 & $44.1 \%$ & 4 & $3.4 \%$ & 19 & $16.1 \%$ & 13 & $11.0 \%$ & 88 & $74.6 \%$ \\
\hline Total & 58 & $49.2 \%$ & 4 & $3.4 \%$ & 25 & $21.2 \%$ & 31 & $26.3 \%$ & 118 & $100.0 \%$ \\
\hline
\end{tabular}

En la Tabla 3, podemos observar, de la población no gestantes el 5.1\% se encontró con pH ácido y el $44.1 \%$ con $\mathrm{pH}$ neutro; el $3.4 \%$ son gestantes de 1 - 14 semanas presentaron pH neutro; gestantes de 15 - 28 semanas presentaron $5.1 \% \mathrm{pH}$ ácido y $16.1 \% \mathrm{pH}$ neutro; gestantes de 29 - 42 semanas, el $15.3 \%$ presentaron pH ácido y el 11\% pH neutro. No se presentó ningún caso con pH alcalino. 
Con un t-student $=5,102 ; \mathrm{gl}=116 ; \mathrm{p}=0.000<0.05$; se puede observar de manera comparativa que las pacientes no gestantes presentaron el mayor porcentaje de $\mathrm{pH}$ neutro, mientras que el mayor porcentaje de pH ácido se encontró en el tercer trimestre de gestación (de 29 - 42 semanas).

Tabla 4. pH salival y flujo salival de gestantes y no gestantes del hospital regional Virgen de Fátima, Chachapoyas - 2018.

\begin{tabular}{|c|c|c|c|c|c|c|c|c|}
\hline \multirow{3}{*}{$\begin{array}{c}\text { pH } \\
\text { salival }\end{array}$} & \multicolumn{6}{|c|}{ Flujo Salival } & \multicolumn{2}{|c|}{ Total } \\
\hline & \multicolumn{2}{|c|}{ Bajo } & \multicolumn{2}{|c|}{ Normal } & \multicolumn{2}{|c|}{ Alto } & \multirow[b]{2}{*}{ fi } & \multirow[b]{2}{*}{$\%$} \\
\hline & $\mathbf{F i}$ & $\%$ & fi & $\%$ & fi & $\%$ & & \\
\hline Acido & 0 & $0.0 \%$ & 0 & $0.0 \%$ & 30 & $25.4 \%$ & 30 & $25.4 \%$ \\
\hline Neutro & 1 & $0.8 \%$ & 3 & $2.5 \%$ & 84 & $71.2 \%$ & 88 & $74.6 \%$ \\
\hline Total & 1 & $0.8 \%$ & 3 & $2.5 \%$ & 114 & $96.6 \%$ & 118 & $100.0 \%$ \\
\hline
\end{tabular}

Fuente: Ficha de recolección de datos.

En la Tabla 4, se puede interpretar lo siguiente: que el $25.4 \%$ de la población que presentó pH ácido presentaba un flujo salival alto. Del $74.5 \%$ de la población que presentó pH neutro, el $71.2 \%$ se encontraba con un flujo salival alto, el $2.5 \%$ con un flujo salival normal y solo el $0.8 \%$ con un flujo salival bajo.

La ji cuadrada $=1,411 ; \mathrm{gl}=2 ; \mathrm{p}=0,494 .>\alpha=0.05$. Nos indica que no existe una relación estadísticamente significativa entre el $\mathrm{pH}$ y flujo salival en gestantes y no gestantes del hospital regional Virgen de Fátima, Chachapoyas - 2018.

Tabla 5. Flujo salival entre gestantes y no gestantes del hospital regional Virgen de Fátima, Chachapoyas - 2018.

\begin{tabular}{|c|c|c|c|c|c|c|c|c|c|c|}
\hline \multirow{3}{*}{$\begin{array}{c}\text { Flujo } \\
\text { Salival } \\
\text { Bajo }\end{array}$} & \multicolumn{8}{|c|}{ Edad gestacional } & & \multirow[b]{2}{*}{ Total } \\
\hline & \multicolumn{2}{|c|}{ No gestante } & \multicolumn{2}{|c|}{$\begin{array}{r}\text { De } 1 \text { a } 14 \\
\text { semanas }\end{array}$} & \multicolumn{2}{|c|}{$\begin{array}{c}\text { De } 15 \text { a } 28 \\
\text { semanas }\end{array}$} & \multicolumn{2}{|c|}{$\begin{array}{r}\text { De } 29 \text { a } 42 \\
\text { Semanas }\end{array}$} & & \\
\hline & 0 & $0.0 \%$ & 0 & $0.0 \%$ & 0 & $0.0 \%$ & 1 & $0.8 \%$ & 1 & $0.8 \%$ \\
\hline Normal & 3 & $2.5 \%$ & 0 & $0.0 \%$ & 0 & $0.0 \%$ & 0 & $0.0 \%$ & 3 & $2.5 \%$ \\
\hline Alto & 55 & $46.6 \%$ & 4 & $3.4 \%$ & 25 & $21.2 \%$ & 30 & $25.4 \%$ & 114 & $96.6 \%$ \\
\hline Total & 58 & $49.2 \%$ & 4 & $3.4 \%$ & 25 & $21.2 \%$ & 31 & $26.3 \%$ & 118 & $100.0 \%$ \\
\hline
\end{tabular}

Fuente: Ficha de recolección de datos.

En la Tabla 5, se puede observar que del $49.2 \%$ que representa a las no gestantes, el $46.6 \%$ se encontró con un flujo salival alto y el $2.5 \%$ normal. Del $50.8 \%$ que representa a las gestantes el $50 \%$ presentó un flujo salival alto y el $0.8 \%$ bajo. 
La ji cuadrada $=5,964 ; \mathrm{gl}=6 ; \mathrm{p}=0,427 .>\alpha=0.05$. Nos indica que no existen diferencias estadísticamente significativas del flujo salival en gestantes y no gestantes del hospital regional Virgen de Fátima, Chachapoyas - 2018.

\section{DISCUSIÓN}

En la Tabla 1, se puede observar que, en relación al pH salival, el $44.1 \%$ presentó pH neutro eran no gestantes y solo el $5.1 \%$ presentaron pH ácido. El $20.4 \%$ que presentó pH ácido y el $30.5 \%$ pH neutro, eran gestantes; esto señala que, existió una disminución significativa del pH salival en gestantes; sin embargo, Ortiz et al. (2012), al evaluar el pH salival de los pacientes gestantes y no gestantes, encontró que el pH de las mujeres embarazadas tenía similitud y eran ligeramente más ácido que el de las mujeres no embarazadas, de esta manera se encontraban en el rango de "pH seguro".

En relación al flujo salival, no existe diferencia significativa entre gestantes y no gestantes, Cerna (2010), indica lo contrario en su estudio, encontró que el flujo salival de las gestantes es más bajo que el grupo de no gestantes.

Con relación del pH salival y el nivel de placa dental entre los trimestres de gestación, existe una diferencia, estableciendo el tercer trimestre como el más afectado durante la gestación; así, Martínez et al. (2014), al realizar las características fisicoquímicas y microbiológicas de la saliva durante y después del embarazo, los resultados fueron similares, pues el $\mathrm{pH}$ y la capacidad amortiguadora de la saliva durante el embarazo fueron bajos que en el posparto, mientras que la tasa de secreción salival fue más alta en el embarazo.

En la Tabla 3, Se puede observar de manera comparativa que las pacientes no gestantes presentaron el mayor porcentaje de $\mathrm{pH}$ neutro, mientras que el mayor porcentaje de $\mathrm{pH}$ ácido se encontró en el tercer trimestre de gestación (de 29 - 42 semanas), por el contrario, Rivasplata (2014), al comparar el pH salival de los gestantes durante los trimestres del embarazo, observó que, no existen diferencias significativas entre los grupos.

Se puede atribuir que los resultados se deben a la técnica incorrecta de cepillado, a la frecuencia con la que se realiza el cepillado y a la dieta de la paciente, que va cambiando por el crecimiento del feto y a la falta de instrucción del personal odontológico.

\section{CONCLUSIONES}

Existe una relación estadísticamente significativa entre el $\mathrm{pH}$ y la placa dental en gestantes y no gestantes del hospital regional Virgen de Fátima, Chachapoyas - 2018.

Existen diferencias estadísticamente significativas del $\mathrm{pH}$ salival entre gestantes y no gestantes del hospital regional Virgen de Fátima, Chachapoyas - 2018. 
El mayor porcentaje de gestantes con $\mathrm{pH}$ ácido, se encontraron en el tercer trimestre de gestación. El mayor porcentaje de gestantes encontradas en un índice malo pertenecen al tercer trimestre de gestación.

\section{REFERENCIAS BIBLIOGRÁFICAS}

Ayala, J. (2008). Determinación del pH salival después del consumo de una dieta cariogénica con y sin cepillado dental previo en niños (Tesis de pregrado). Universidad Nacional Mayor de San Marcos, Lima, Perú.

Bazan, D. (2016). CPOD, volumen de flujo salival y nivel de $\mathrm{pH}$ salival en adolescentes gestantes y no gestantes del hospital de segundo nivel de barranca - Cajatambo. Lima: Dirección Regional de Salud.

Beltran, A. (2014). Relación del pH salival con el índice de caries dental e índice periodontal en pacientes geriátricos del asilo de ancianos Víctor Lira, Arequipa (tesis de pregrado). Universidad Católica Santa María de Arequipa, Perú.

Beltran, V. (2018). Características del cepillado dental en mujeres embarazadas que acuden a la clínica humanitaria fundación Pablo Jaramillo Crespo (Tesis de pregrado). Universidad de Cuenca, Ecuador.

Canales, F. (1994). Metodología de la investigación Científica. Manual para el desarrollo de personal de salud. México: Editorial Washington.

Caridad, C. (2008). El pH, flujo salival y capacidad buffer en relación a la formación de la placa dental. $\begin{array}{llll}\text { Odous } & \text { científica. } & \text { IX. }\end{array}$ http://servicio.bc.uc.edu.ve/odontologia/revista/v9n1/art3.pdf

Castillo, J. (2009). Capacidad buffer, Flujo y pH salival en Gestantes del último trimestre, puerperio y no gestantes que acuden al centro de salud materno infantil Santa Isabel. El Porvenir (tesis de pregrado). Universidad Nacional de Trujillo, Perú.

Cerna, E. (2010). PH Y Flujo Salival En Gestantes Del Primer Trimestre De Embarazo Procedentes Del Hospital "María Auxiliadora", Distrito De San Juan De Miraflores, Lima-2010 (tesis de pregrado). Universidad Privada Norbert Wiener, Lima.

Chamilco, A. (2013). Variación del PH y flujo salival durante el periodo gestacional en embarazadas de un servicio asistencial público, tesis de pregrado. Lima.

Chaupis, I. (2016). Variación del pH y flujo salival durante el periodo gestacional para evaluar el riesgo estomatológico en el Hospital Militar Central Lima 2016 (Tesis de pregrado). Universidad de Huánuco. 
http://repositorio.udh.edu.pe/bitstream/handle/123456789/95/Chaupis_D\%C3\%A

1vila_ingirid_tesis_titulo_2016.pdf?sequence=1\&isAllowed=y

Cosio, D., Ortega, A., \& Vaillard, D. (2010). Determinación del Ph salival antes durante y después del consumo de caramelos en niños y niñas de 3, 4,5 años de edad, Oral, 11(35), 642-645.

Delgado, K, (2017). Caries dental relacionado con el pH salival en adolescentes de una institución educativa del distrito de Paiján - Ascope, 2016 (Tesis de Pregrado). Universidad Privada Antenor Orrego, Trujillo.

Echeverri, M. (1995). La Saliva, componentes, función y Patología. Revista Estomatológica de Cali, 4(2); 5 (1), 1- 104.

Gómez, M. E., y Campos, A. (2019). Histología, embriología e ingeniería tisular bucodental. Panamericana

Finlay, C. (2010). Elementos necesarios sobre salud bucal. Revista Cubana Estomatológica; 44(4), 21 24.

Góngora, C. y Puerta, I., (2014). Relación entre el pH salival y caries dental en pacientes con VIH del programa TARGA del Hospital Regional de Loreto, 2014 (Tesis de pregrado). Universidad Nacional de la Amazonía Peruana, Perú.

Gonzales, M., Montes, L. y Jiménez, G. (2001) Cambios en la composición de la saliva de pacientes gestantes y no gestantes (Tesis de pregrado). Universidad Autónoma del Estado de México.

Guevara, P. (2017). Efecto del consumo de bebidas envasadas en la variación del pH salival en niños de la institución educativa inicial Raquel robles de Román, Chachapoyas - 2017 (Tesis de pregrado). Universidad Nacional Toribio Rodríguez de Mendoza de Amazonas, Perú.

Gutiérrez, J. (2013). Comparar el nivel de pH salival en las diferentes etapas de la enfermedad periodontal (Tesis de posgrado). Universidad Autónoma de Nuevo León, México.

Gutiérrez, M., Ortiz, L., Medina, K. y Chein, S. (2007). Eficacia de una medida preventiva para el niño con riesgo cariogénico asociada a la estabilidad de ph salival. Odontología San Marquina, 10 (1), 25-27.

Jiménez, R. (2004). Importancia del pH, flujo y viscosidad salival sobre el desarrollo de caries dental en mujeres gestantes del primer trimestre (Tesis de pregrado). Universidad Nacional Mayor de San Marcos, Lima.

Limo, L. (2017). El IHOS y el CPOD y su relación con el pH salival en alumnos con habilidades diferentes con retardo mental del centro ANN Sullivan del Perú. KIRU, 14 (1), 35-45. DOI: org/10.24265/KIRU. 2017.v14.

Martínez, C. (2008). "Exploración de significados con respecto a la Salud Bucal de un Grupo de Gestantes”. Rev. Facultad de Odontología Universidad de Antioquia. 23(1), 76-91. 
Martínez, M., Martínez, C., López, A., Patiño, L. \& Arango, E. (2014). Características fisicoquímicas y microbiológicas de la saliva durante y después del embarazo, journal of dental education. Vol 65.

Medina, J. (2005). Prevalencia de caries y repercusiones estéticas en niños de la institución educativa Juan Pablo del Cono Norte (Tesis de maestría). Universidad Nacional Mayor de San Marcos, Lima.

Sánchez, A. L., y Gonzáles, E. (2016). Prevalencia de caries dental en escolares de nivel primario de la Institución Educativa 18006 Pedro Castro Alva, Chachapoyas, 2017. Revista de Investigación científica UNTRM, 1(3), 20-25.

Misrachi, C. (2010). Un modelo para la promoción de la salud focalizado en la escuela y su operacionalización. "Un modelo para la promoción de la salud focalizado en la escuela y su operacionalización"; enfoques en la atención primaria, 5 (4), 7 - 14.

Ministerio de Salud. (2015). Salud bucal-índices de caries en el Perú, [fecha de consulta: 22 diciembre 2019]. Disponible en: http:www.minsa.gob.pe.

Organización mundial de la salud - OMS (2018). Enfermedades orales, recuperado de https://www.oms/enforales/2018/.

Ortiz, D., Olvera, A., Carreón, C. G., y Bologna, C. R. (2012). Evaluación del pH salival en pacientes gestantes y no gestantes. Rev. ADM., 69(3), 125-130.

Purizaca, M. (2010). Modificaciones fisiológicas en el embarazo. Revista peruana de Ginecología y Obstetricia. 56 (1), 57-69.

Puscan, J. (2017). Frecuencia de caries dental en primeros molares permanentes en estudiantes de la Institución Educativa $\mathrm{N}^{\circ} 18040$ de Huancas (Tesis de pregrado). Universidad Nacional Toribio Rodríguez de Mendoza de Amazonas, Perú.

Rivasplata, I. (2014). Comparación Del pH Salival En Gestantes Durante Los Trimestres Del Embarazo En El Hospital Belén De Trujillo (Tesis de pregrado). Universidad Nacional de Trujillo, Perú.

Rojas, T., Romero, M., Navas, R., Álvarez, C. y Morón, M. (2008). Flujo salival, ph y capacidad amortiguadora en niños cardiópatas: factor de riesgo para caries dental y enfermedad periodontal. Estudio preliminar. Ciencia Odontológica. 5(1), 17-26.

Romero, M. y Hernández, Y. (2009). Modificaciones del pH y flujo salival con el uso de aparatología funcional tipo Bimler. Revista latinoamericana de ortodoncia y ortopedia, Ortodoncia. Disponible en https://www.ortodoncia.ws/publicaciones/2009/art-6/...

Supo, J. (2014). Metodología de la Investigación científica. 5ta. Edic. Arequipa: Edit. Universitaria. 
Tricerri, P. (2015). PH salival y su relación con la caries/gingivitis de las gestantes atendidas en el Centro de Salud Atención 24 Horas Andrés de Vera, período marzo - julio (Tesis de grado). Universidad San Gregorio, Portoviejo.

Thylstrup, A. (1988). Caries Dental, Ediciones Doyma, pp. 154-155.

Vargas, D. (2015). Estudio del pH salival en relación a la placa bacteriana en niños de 7 a 12 años atendidos en la escuela Teresa Flor (Tesis de pregrado). Universidad de Guayaquil, Ecuador. 